\title{
Effects of compassion training on brain responses to suffering others
}

\author{
Yoni K. Ashar,(1,2 Jessica R. Andrews-Hanna, ${ }^{3}$ Joan Halifax, ${ }^{4}$ \\ Sona Dimidjian, ${ }^{5,6}$ and Tor D. Wager ${ }^{7}$
}

${ }^{1}$ Department of Psychology and Neuroscience, University of Colorado Boulder, Boulder, CO 80309, USA, ${ }^{2}$ Weill Cornell Medical College, New York, NY 10075, USA, ${ }^{3}$ Department of Psychology, University of Arizona, Tucson, AZ 85721, USA, ${ }^{4}$ Upaya Institute and Zen Center, Santa Fe, NM 87501, USA, ${ }^{5}$ Department of Psychology and Neuroscience, University of Colorado Boulder, Boulder, CO 80309, USA, ${ }^{6}$ Renee Crown Wellness Institute, University of Colorado Boulder, Boulder, CO 80309, USA, and ${ }^{7}$ Department of Psychology and Brain Sciences, Dartmouth College, Hanover, NH 03755, USA

Correspondence should be addressed to Tor D. Wager, Department of Psychology and Brain Sciences, Dartmouth College, HB 6207, Hanover, NH 03755, USA. E-mail: tor.d.wager@dartmouth.edu.

\begin{abstract}
Compassion meditation (CM) is a promising intervention for enhancing compassion, although its active ingredients and neurobiological mechanisms are not well-understood. To investigate these, we conducted a three-armed placebo-controlled randomized trial $(\mathrm{N}=57)$ with longitudinal functional magnetic resonance imaging (fMRI). We compared a 4-week $\mathrm{CM}$ program delivered by smartphone application with (i) a placebo condition, presented to participants as the compassionenhancing hormone oxytocin, and (ii) a condition designed to control for increased familiarity with suffering others, an element of CM which may promote compassion. At pre- and post-intervention, participants listened to compassioneliciting narratives describing suffering others during fMRI. CM increased brain responses to suffering others in the medial orbitofrontal cortex (mOFC) relative to the familiarity condition, $p<0.05$ family-wise error rate corrected. Among CM participants, individual differences in increased mOFC responses positively correlated with increased compassion-related feelings and attributions, $r=0.50, p=0.04$. Relative to placebo, the CM group exhibited a similar increase in mOFC activity at an uncorrected threshold of $P<0.001$ and 10 contiguous voxels. We conclude that the $\mathrm{mOFC}$, a region closely related to affiliative affect and motivation, is an important brain mechanism of CM. Effects of CM on mOFC function are not explained by familiarity effects and are partly explained by placebo effects.
\end{abstract}

Key words: compassion training; empathy; mindfulness; placebo; burnout

\section{Introduction}

Compassion is a vital societal and interpersonal process that facilitates caring and cooperative behavior (Goetz et al., 2010) and is widely recognized as a virtue across religious and ethical frameworks. Recently, scientific interest has turned toward the cultivation of compassion. Evidence is accumulating that compassion-focused interventions yield benefits for the self and others in community samples (Galante et al., 2014; Quaglia et al., 2020), mitigate burnout in medical providers (Van Berkhout and Malouff, 2015) and improve symptoms and functioning in several patient populations (Hofmann et al., 2011; Gilbert, 2014; Darnall, 2015; Shonin et al., 2015; Berry et al., 2020).

Received: 30 August 2019; Revised: 7 April 2021; Accepted: 4 May 2021

(C) The Author(s) 2021. Published by Oxford University Press.

This is an Open Access article distributed under the terms of the Creative Commons Attribution License (https://creativecommons.org/licenses/by/4.0/), which permits unrestricted reuse, distribution, and reproduction in any medium, provided the original work is properly cited. 
Many compassion training programs have been based on compassion meditation (CM), a secular meditation practice with Buddhist origins. In the scientific literature, $\mathrm{CM}$ has been viewed as a practice that influences appraisals of suffering others and consequent emotional responses (Ashar et al., 2016a; Weng et al., 2017), leading to increased empathic care, vicarious optimism, tenderness and other positive (but not negative) affective responses to suffering others (Klimecki et al., 2012; Galante et al., 2014; Ashar et al., 2016b; Kok et al., 2016; Koopmann-Holm et al., 2020; Sirotina and Shchebetenko, 2020). CM-based interventions have also been found to promote a range of helping behaviors such as charitable donations and offering one's seat to a suffering person on crutches (Condon et al., 2013; Weng et al., 2013, 2015; Lim et al., 2015; Ashar et al., 2016b; Böckler et al., 2018). At the biological level, CM-based interventions have been found to reduce inflammation (Pace et al., 2009, 2012) and enhance parasympathetic activity (Bornemann et al., 2016), suggesting potential health benefits as well.

Multiple distributed brain systems are known to support empathy, compassion and altruism. These span cortical networks and subcortical structures that enable inferences about others' mental states, simulation of other's embodied experiences, the valuing of others' welfare and more (De Waal, 2008; Shamay-Tsoory et al., 2009; Decety et al., 2012; Zaki and Ochsner, 2012). Previous investigations of CM have highlighted a key role for two brain systems in particular. One line of research has consistently found that CM engages a mesolimbic pathway, including the nucleus accumbens (NAc), ventral tegmental area (VTA) and medial orbitofrontal cortex (mOFC). These regions show a heightened response to suffering others following a CM intervention, are engaged by compassionate reappraisal of suffering and exhibit cortical thickening among expert CM practitioners (Klimecki et al., 2014; Singer and Klimecki, 2014; Engen and Singer, 2015b; Engen et al., 2018). This pathway is closely tied to positive affect, value and motivation (Haber and Knutson, 2010; Berridge and Kringelbach, 2015) and more broadly to the use of conceptual processes to assign personal meaning to events (Roy et al., 2012; Barrett, 2017; Ashar et al., 2017b). In the interpersonal context, this system has been linked to empathic care, affiliation, interpersonal closeness and vicarious reward (Koenigs et al., 2007; Mobbs et al., 2009; Hare et al., 2010; Krienen et al., 2010; Inagaki and Eisenberger, 2012; Meyer et al., 2013; Moll et al., 2014; Morelli et al., 2015; Ashar et al., 2017a). This suggests that CM may enhance compassion by increasing the value placed on others' welfare and/or by increasing affiliative affect, perceived interpersonal closeness and related processes (Goetz et al., 2010; Zickfeld et al., 2019).

In distinction, other studies have reported effects of $\mathrm{CM}$ primarily in the dorsomedial prefrontal cortex (dmPFC) and temporal-parietal junction (TPJ), including heightened responses to suffering others and cortical thickening following CM-based interventions (Lutz et al., 2008; Mascaro et al., 2013; Weng et al., 2013; Valk et al., 2017; Favre et al., 2020). These regions-key components of the default mode networksupport perspective-taking and inferences about others' internal states (Saxe and Kanwisher, 2003; Buckner and Carroll, 2007; Tankersley et al., 2007; Shamay-Tsoory et al., 2009; Zaki and Ochsner, 2012), suggesting that CM may enhance compassion by promoting these and related processes.

Based on these two sets of findings, a recent debate has focused on whether CM primarily acts on affective or cognitive processes (Dahl et al., 2015, 2016; Engen and Singer, 2015a)-although these likely interact to support compassion (Kanske et al., 2016). We thus focused our analyses on these two sets of regions, to better understand how CM engages brain pathways more closely related to affiliation and affect us those more closely related to mentalizing and perspective-taking.

A central challenge in the study of $\mathrm{CM}$ has been identifying and controlling for 'non-specific' factors such as engagement in a regular practice, familiarity with and attention to suffering others and expectations of heightened compassion. While a recent large-scale study found specific effects of CM vs other meditation practices on several compassion-related outcomes (Klimecki et al., 2014; Kok and Singer, 2017; Singer and Engert, 2019), other studies have found relatively weak support for this, showing little to no difference between $\mathrm{CM}$ and active control on compassion-related outcomes (Condon et al., 2013; Galante et al., 2014; Kreplin et al., 2018; Koopmann-Holm et al., 2020). A potentially important, understudied factor is the meditator's expectations of increased compassion-and in the laboratory context, perceived researcher expectations of increased compassion (demand characteristics). These processes are known to influence empathy and helping behaviors (Rutgen et al., 2015; Rütgen et al., 2015; Nook et al., 2016), but it is unclear whether commonly used control conditions for CM (e.g. mindfulness meditation interventions) are matched on expectations of increased compassion. Another non-specific element of CM is the increased familiarity with suffering others created by meditating on them during CM practice. This might increase awareness of suffering others or influence preferences toward familiar individuals, leading to increase compassion (Zajonc, 2001; Loewenstein and Small, 2007). Comparison conditions controlling for expectations, demand characteristics and increased familiarity with suffering others are needed to better understand the 'active ingredients' of CM.

Here, we conducted a three-armed placebo-controlled randomized controlled trial of $\mathrm{CM}$, with each group receiving a 4-week intervention delivered daily by a mobile iPod application. The CM program aimed to teach participants skills for staying engaged with others' suffering without becoming emotionally overwhelmed (Halifax, 2011, 2012), since engaging with others' suffering can tax cognitive and emotional resources (Zaki, 2014; Cameron, 2017; Cameron et al., 2019). A comparison group received a placebo intervention-a nasal spray described to them as the compassion-enhancing hormone oxytocinalthough it was actually saline. A third group simply listened to brief narratives of suffering others daily and answered factual questions. To investigate the neurobiological effects of $\mathrm{CM}$, we collected functional magnetic resonance imaging (fMRI) preand post-intervention.

Intervention effects on self-reported and behavioral outcomes from this trial have been previously reported (Ashar et al., 2016b). The goal of this article was to test the effects of $\mathrm{CM}$ on the brain regions of interest described above, with a secondary goal of testing $\mathrm{CM}$ effects on recently developed multivariate patterns of brain function related to empathic emotions (Ashar et al., 2017a), described further below.

\section{Method}

\section{Participants}

Out of 311 participants screened for eligibility, 71 healthy adults completed the baseline assessment between January and September of 2012. To be eligible, participants were required to self-report no history of major psychiatric illness, current mental health conditions or breast-feeding (to maintain the oxytocin placebo deception). Standard fMRI exclusion criteria were 
applied (e.g. no metal in the body and no claustrophobia). Participants were also required to have no previous experience with CM or Loving-Kindness Meditation and at least moderate interest in meditation, as we sought to investigate the effects of CM among healthy, interested novices (e.g. see also Segal et al., 2010; Williams et al., 2014). We also excluded participants who reported in advance that they would not be willing to donate any participation earnings to charities. An additional research aim of ours, orthogonal to the questions addressed in the present manuscript, was to identify within-person behavior and brain predictors of charitable donations (results described in Ashar et al., 2016b, 2017a). Since this requires within-person variability in donation amounts, we excluded participants likely to have no variance in this measure.

Thirteen participants who completed the baseline assessment were not eligible for randomization for a variety of technical reasons (excessive head motion during the baseline fMRI scan: $n=7$; lost to follow-up: $n=2$; no donations made: $n=2$; neurological anomaly discovered at baseline scan: $n=1$ and not interested in continuing the study, $n=1$ ). Thus, $N=58$ participants were randomized using a computer-generated randomization list, stratified by sex. One participant refused randomization to the placebo oxytocin condition due to unwillingness to use a nasal spray. Thus, the final sample included $N=57$ analyzed participants, including $N=36$ females, with $\mathrm{M}_{\text {age }}=29.11$ years, $\mathrm{SD}_{\text {age }}=6.35$ years, $\mathrm{M}_{\text {Subjective } \mathrm{SES}}=6.25$ out of 10 and $\mathrm{SD}_{\text {Subjective SES }}=1.65$. Subjective Socioeconomic Status (SES) was measured with a single-item measure (Adler et al., 2000). Additionally, one participant misunderstood the donation task instructions and was dropped from analyses of the charitable donation outcomes. Experimenters were blind to the participants' assigned intervention for the pre-intervention assessment but not for the post-intervention assessment. Participant demographics and baseline characteristics are provided for each intervention condition in Table 1. Sample size was dictated by the available research funds.

Participants were compensated $\$ 100$ for each MRI session and an additional $\$ 1$ for each day of the intervention that they completed. After completion of the study, participants in the placebo condition completed a questionnaire assessing the strength of their belief that they were actually taking oxytocin and were then debriefed regarding the nature of the deception and its purpose. The University of Colorado Institutional Review Board approved all procedures, including informed consent. No serious adverse events resulted from any of the intervention conditions.

Table 1. Participants' demographics

\begin{tabular}{llll}
\hline & CM & OxyPla & Familiarity \\
\hline Sample size $(n)$ & 21 & 18 & 18 \\
\hline $\begin{array}{c}\text { Sex: } n \text { female } \\
\text { (\% female) }\end{array}$ & $14(67)$ & $11(61)$ & $11(61)$ \\
$\begin{array}{c}\text { Age (years): } \\
\text { M (SD) }\end{array}$ & $28.72(6.83)$ & $27.43(4.02)$ & $29.63(7.45)$ \\
$\begin{array}{c}\text { Subjective SES } \\
\text { Race: } n \text { White } \\
\text { (\% White) }\end{array}$ & $6.10(1.71)$ & $6.24(1.56)$ & $6.65(1.50)$ \\
& & $15(83)$ & $14(78)$ \\
\hline
\end{tabular}

Subjective socioeconomic status (SSES) was measured by the MacArthur Scale of SSESS, a 10-point Likert scale ranging from lowest to highest SES (Adler et al., 2000).

\section{Materials and procedures}

Brain responses to suffering others. To assess compassionrelated brain function, we designed a task resembling dailylife encounters with suffering others. During fMRI scanning conducted before and after the 4-week intervention, participants listened to 24 randomly ordered biographies describing a range of true stories of suffering people such as orphaned children, adults with cancer and homeless veterans. To increase ecological validity, biographies were created from factual information posted on charity websites and then were recorded by one member of the research team as audio segments 26-33.5 $\mathrm{s}$ in duration. An authentic facial photograph of each person, also drawn from the charity website, was displayed alongside the audio biography. The people described in the biographies were balanced on age (child or adult), race (Black or White) and sex. Real stories and photographs were used to increase ecological validity. An example biography is: 'Jessica's father abandoned his family and her mother was unable to support them alone, so they had to move into a homeless shelter. The shelter provided Jessica's mother with professional training and childcare. Eventually, the family moved into subsidized housing. Jessica and her sisters have been tremendously supportive of each other. Jessica has managed to stay in school and will finish the year with her class'. To hear this biography while viewing a sample face photograph, as presented to our subjects, visit https://canlabweb.colorado.edu/files/jessica.mp4 (image copyright CC BY-SA 2.0). The text of all biographies is listed in Supplementary Table S1 of Ashar et al., (2017a), and audio and video recordings of all biographies are available for download at https://github.com/canlab/Paradigms_Public/tree/ master/2016_Ashar_Empathy_CompassionMeditation. After each biography, participants provided ratings of empathic care or empathic distress (data not presented here). Given the limited time during functional imaging, we collected ratings of other relevant feelings and attributions immediately after the scan, described below.

After this first task in the scanner, participants then heard abbreviated 'reminder' biographies (8-11s) during fMRI scanning while viewing the face photograph of that person. An example reminder is: 'Jessica's father abandoned his family. Her mother and sisters moved into a homeless shelter, which provided job training and childcare. Jessica will finish school this year with her class'. Reminders were provided because pilot studies showed that participants had difficulty distinctly recalling each of the 24 biographies when asked to make donation decisions. Following each reminder, participants were given an option to donate a portion of their own experimental earnings to a charity helping that person, from $\$ 0$ to $\$ 100$ in $\$ 1$ increments, as a measure of compassionate behavior. Between trials, participants were asked to press a button indicating the direction an arrow was pointing (left or right); this served as a non-social baseline comparison task. The duration of this inter-trial baseline task was jittered across trials, from 3 to $9 \mathrm{~s}$. During the task, participants were asked to simply listen to the biographies, and $\mathrm{CM}$ participants at the post-intervention assessment were asked not to engage in $\mathrm{CM}$ while listening to the biographies, for greater comparability across conditions.

This task was completed over three fMRI runs of listening to biographies and rating feelings, followed by two runs of listening to biography reminders and making donations. The task is publicly available at https://github.com/canlab/Paradigms_Public/ tree/master/2016_Ashar_Empathy_CompassionMeditation. 
Self-reported and behavioral measures of compassion. Primary self-reported and behavioral outcomes were charitable donations, as described in the fMRI task above, and compassion-related feelings and attributions. These ratings included attributions of blame-worthiness for one's suffering, attributions of how much a person would be benefitted by efforts to help them, feelings of distress and tenderness and perceived similarity both in socioeconomic status and in values and interests. In prior work (Study 1 in Ashar et al., 2016b), we reported that a linear combination of these feelings and attributions, termed 'Feeling-Attribution-Similarity' (or FAS) scores, was strongly predictive of charitable donation. We applied this model to the data collected in this study, to generate FAS scores for each participant at pre- and postintervention. Participants provided ratings on these feelings and attributions on a visual analog scale ranging from 'not at all' to 'extremely'. These ratings were provided after listening to the biographies a second time after scanning, as there was insufficient time during scanning to collect all ratings on all stimuli.

Interventions. After the baseline session, participants were randomized to one of the three interventions-Familiarity, Placebo Oxytocin (OxyPla) or CM training-with $N_{\mathrm{CM}}=21, N_{\text {OxyPla }}=18$ and $N_{\text {familiarity }}=18$. The three interventions were delivered via iPod Touch applications developed by the study team and matched across conditions on structure and style. All participants were asked to complete a daily task for 4 weeks on the iPod Touch provided to them. A member of the study team placed three phone calls to participants during the intervention to address any concerns, ask about side effects in the OxyPla condition and encourage compliance.

Participants in all three conditions listened to a biography of a suffering person every day while viewing a photograph of that person. Out of the 24 total biographies presented during the fMRI sessions, each participant listened to and viewed a set of 12 biographies across the 4-week intervention period. The set of biographies presented to each participant during the intervention was randomly assigned and balanced across groups.

Compassion meditation. The CM program was designed to enhance both compassion for suffering others and equanimity. The emphasis on equanimity aimed to help prevent emotional overwhelm from others' suffering, thereby promoting sustainable compassionate responding (Halifax, 2012). A theme of the meditation recordings was 'soft front, strong back', which served as both a metaphor and an embodied approach to being sensitive to and engaged with others' suffering while remaining emotionally grounded. Meditations included a focus on grounding in the body and connection with the earth as a foundation, perspective-taking practices (e.g. imagine what this person may be experiencing), visual imagery (e.g. imagine the suffering person as a small child and tong-len practice) and repetition of compassion-related phrases directed toward the suffering person (e.g. 'may you find peace'). The meditations also asked participants to direct these practices toward loved ones, for whom it is often easier to generate compassion, and toward themselves, to enhance self-compassion. Participants were asked to practice meditation for about 20 min daily and were provided with a new guided meditation practice at the start of each of the 4
Table 2. Description of the compassion meditation program

\begin{tabular}{ll}
\hline Week & Elements \\
\hline $\begin{array}{l}\text { Elements } \\
\text { present in } \\
\text { all weeks }\end{array}$ & $\begin{array}{c}\text { Grounding in mindful awareness of breath and } \\
\text { body sensations. The refrain 'soft front, strong } \\
\text { back' as an embodied metaphor for equanimity } \\
\text { and compassion } \\
\text { Week 1 }\end{array}$ \\
& $\begin{array}{l}\text { Generate compassion for oneself, a close other } \\
\text { and the person described in the story using short } \\
\text { phrases (e.g. 'may you be safe' and 'may you be free } \\
\text { from suffering') }\end{array}$ \\
Week 2 & $\begin{array}{l}\text { Imagine yourself as a young, happy child, innocent } \\
\text { and blameless. Imagine a suffering other as a }\end{array}$ \\
& $\begin{array}{l}\text { young, happy child, innocent and blameless, to } \\
\text { generate compassion }\end{array}$ \\
Week 3 & $\begin{array}{l}\text { Take the perspective of a close other who is suffer- } \\
\text { ing. Imagine their history of lived experience and } \\
\text { the current difficulties they may be experiencing }\end{array}$ \\
Week 4 & $\begin{array}{l}\text { Tong-len. Breathe in the suffering of another person, } \\
\text { visualized as hot smoky air. Exhale relief and heal- }\end{array}$ \\
ing back to the suffering person, visualized as cool \\
clean air
\end{tabular}

weeks. At a specified point during each daily meditation, participants would hear one of the biographies described in the fMRI task above and were asked to meditate on that person specifically. A description of each weekly CM practice is provided in Table 2. The meditation scripts and recordings are publicly available at https://github.com/canlab/Paradigms_Public/tree/ master/2016_Ashar_Empathy_CompassionMeditation/CM_aud io_scripts.

Placebo oxytocin intervention. The OxyPla intervention was designed to control for placebo effects related to CM, such as (i) participant expectations of increased compassion naturally created by $\mathrm{CM}$ and (ii) demand characteristics created by completing a CM intervention in a research context (e.g. wanting to satisfy perceived researcher objectives by exhibiting increased compassion). Every day, participants were instructed to inhale a nasal spray labeled as oxytocin, press a button in the smartphone application confirming they did so and then listen to the daily biography. Participants were also provided with scientific information sheets describing oxytocin's ability to enhance compassion. Both the OxyPla and Familiarity conditions required $\sim 2 \mathrm{~min}$ each day. The OxyPla intervention materials, including information sheet and nasal spray bottle labels, are publicly available at https://github.com/canlab/Paradigms_ Public/tree/master/2016_Ashar_Empathy_CompassionMeditati on/placebo_oxytocin_materials.

Familiarity intervention. Participants in the Familiarity condition simply listened to one biography of a suffering person daily. This condition was designed to control for the increased familiarity with suffering others inherent in the CM practice, as familiarity with suffering people could increase liking and enhance compassion (Zajonc, 2001; Loewenstein and Small, 2007). Alternately, this intervention could be viewed from a lens of repeated exposure, which may dampen emotional reactivity over time. 
Daily attention-to-task check. After each daily intervention task, participants in all conditions responded to a multiplechoice question designed to test whether they had adequately paid attention to the biography. Participants were asked to indicate the primary hardship afflicting the individual described in the biography they had heard that day (e.g. 'What was Robert's primary hardship? (i) AIDS, (ii) cancer or (iii) homelessness'). Participants also provided ratings of mood each day (data not presented here).

\section{Analyses}

fMRI data acquisition and preprocessing. Images were acqu ired with a 3.0T Siemens Trio Tim MRI scanner using a 12-channel head coil. Twenty-six $3.0 \mathrm{~mm}$-thick slices (inplane resolution $3.4 \times 3.4 \times 3.0,1 \mathrm{~mm}$ gap, ascending sequential acquisition) extended axially from the mid-pons to the top of the brain, providing whole-brain coverage (Repetition Time $(\mathrm{TR})=1.3 \mathrm{~s}$, Echo Time $(\mathrm{TE})=25 \mathrm{~ms}$, flip $=75^{\circ}$, field of view $=220 \mathrm{~mm}$, matrix size $=64 \times 64 \times 26$ ). High-resolution structural scans were acquired prior to the functional runs with a T1-weighted MP RAGE pulse sequence (TR $=2530 \mathrm{~ms}$, $\mathrm{TE}=1.64 \mathrm{~ms}$, flip $=7^{\circ}$, 192 slices, $1 \times 1 \times 1 \mathrm{~mm}$ ). Parallel image reconstruction (GRAPPA) with an acceleration factor of 2 was used.

Before fMRI preprocessing, volumes were identified as outliers on signal intensity using Mahalanobis distances (3 s.d.), and dummy regressors were included as nuisance covariates in the first-level models. Functional images were corrected for differences in the acquisition timing of each slice and were motion-corrected (realigned) using SPM8. Twentyfour head motion covariates per run were entered into each first-level model (displacement in six dimensions, displacement squared, derivatives of displacement and derivatives squared). Structural T1-weighted images were then coregistered to the mean functional images using SPM8's iterative mutual information-based algorithm. Coregistered, high-resolution structural images were warped to Montreal Neurologic Institute (MNI) space (avg152T1.nii); these warping parameters were applied to the functional data, normalizing it to MNI space, and interpolated to $2 \times 2 \times 2 \mathrm{~mm}^{3}$ voxels. Finally, functional images were smoothed with an $8 \mathrm{~mm}$ FWHM Gaussian kernel. A 220 s high-pass filter was applied during the first-level analysis.

fMRI analyses. Our analyses focused on the period of listening to biography reminders, which were briefer $(11.5 \mathrm{~s})$ than the initial biography listening period (33.5s) and more proximal to charitable donation decisions. We estimated a general linear model using SPM8 for each participant, including the nuisance covariates generated in preprocessing and two regressors of interest: listening to biography reminders $(11.5 \mathrm{~s})$ and the charitable donation decision period ( $5 \mathrm{~s}$ ), each convolved with the standard hemodynamic response function. The jittered-duration inter-trial interval served as the model intercept. We then computed contrast images for the (listen-baseline) comparison for every subject at preand post-intervention. We subtracted the pre-intervention image from the post-intervention image to estimate pre-topost-intervention changes in brain responses to stories of suffering.

We tested two comparisons-CM us Familiarity and CM us OxyPla-to identify the specific effects of CM on brain activity.
For archival purposes, we also estimated the OxyPla us Familiarity comparison to characterize placebo effects on brain activity; these results are reported in the supplementary material (Supplementary Table S1, Figure S6).

Region of interest selection. Previous CM studies have reported effects primarily in two sets of brain regions: (i) elements of networks involved in regulation of motivated behavior, including the OFC, VTA and NAc (Klimecki et al., 2014; Singer and Klimecki, 2014; Engen and Singer, 2015b), which are also part of the mesolimbic dopaminergic pathway, and (ii) regions linked to construction of conceptual models and mentalizing, including the dmPFC and the TPJ (Lutz et al., 2008; Mascaro et al., 2013; Weng et al., 2013; Ashar et al., 2016a; Valk et al., 2017). VTA and NAc definitions were taken from a high-resolution subcortical atlas (Pauli et al., 2018). A mask for the OFC was adopted from a recent multimodal cortical parcellation (region 'OFC' in Glasser et al., 2016). Masks for the dmPFC and TPJ were created from $10 \mathrm{~mm}$ spheres around peak coordinates reported in prior CM studies, with symmetrical (mirrored) spheres in both hemispheres (Mascaro et al., 2013 for dmPFC [-9, 50, 37]; Weng et al. (2013) for TPJ [46, -62, 36]).

We tested for group differences in two masks, one covering each set of regions, at a threshold of $P<0.05$ familywise error rate (FWER) corrected. Correction was performed using permutation testing (10 000 permutations) in FSL randomize v2.9 using threshold-free cluster-enhancement (Smith and Nichols, 2009). Given a priori directional hypotheses from prior literature, we tested for CM us control increases (not decreases) in brain responses to suffering others. In clusters showing a significant group difference, we conducted a robust regression within CM participants testing whether change in brain activity correlated with (i) changes in FAS scores and (ii) changes in charitable donations.

Whole-brain gray matter analyses. To more broadly characterize intervention effects on brain function, we conducted a whole-brain robust regression (Wager et al., 2005) estimating group differences in pre-to-post-intervention changes in brain activity. We also conducted a robust regression estimating average pre-to-post-intervention changes within each group, to characterize absolute pre-to-post-intervention changes independently for each group. Analyses were conducted two-tailed within a gray matter mask, as results in white matter and cerebrospinal fluid are unlikely to reflect changes in neuronal activity. As part of a standard quality-control process, we also verified that there were no unexpected regions of activation in white matter and ventricle spaces that would be consistent with artifacts. We applied an exploratory threshold of $P<0.001$ uncorrected and 10 or more contiguous voxels. This is a commonly used threshold providing a balance between type I and type II error rates. It is most appropriate for hypothesisgeneration purposes and aggregation of findings across studies in meta-analyses, especially in whole-brain analyses with limited sample sizes and moderately sized effects (Lieberman and Cunningham, 2009; Woo et al., 2014).

Neuroanatomical labeling was conducted using the freely available CanlabCore tools, which pool anatomical labels from across number of published atlases (see https://canlabcore. readthedocs.io/en/latest/moduleslist.html\#@region.table) and with reference to the a recent histological atlas (Amunts et al., 2020). Contrast images for each subject at each time point, behavioral outcomes and code for all analyses are publicly available at https://github.com/yonestar/effects_of_CM_on_brain. 
Intervention effects on brain models of empathic care and distress. We tested the effect of the interventions on two previously published whole-brain patterns associated with two distinct emotional responses to suffering others: empathic care-an affiliative, tender emotional response-and empathic distress, a high-arousal negatively valenced emotional response (Ashar et al., 2017a). We hypothesized that the CM group would exhibit pre-to-post-intervention increases in the empathic care brain model relative to control conditions. A secondary hypothesis was of increases in the empathic distress brain model for CM us Familiarity, driven primarily by decreased distress for Familiarity participants, potentially paralleling the previously published behavioral outcomes.

We computed the cosine similarity between the care and distress models and each subjects' contrast images at both pre- and post-intervention. Cosine similarity is a normalized measure of similarity between two vectors (i.e. brain patterns). It is equivalent to both (i) the dot product after normalizing whole-brain image intensity and (ii) Pearson's correlation without meancentering images and can be used for assessing multivariate pattern expression in test images (e.g. Mitchell et al., 2008; Kragel et al., 2018). We computed a pre-to-post-intervention change score for each participant for both the empathic care and empathic distress models and submitted these to twosample T-tests of CM us OxyPla and CM us Familiarity group differences.

Tests of CM protecting against decreased brain responses to suffering others. In the behavioral outcomes, we observed significant, unexpected decreases in the Familiarity group for compassion-related feelings and attributions (measured with FAS scores) and for charitable donations (Figure 1B). CM protected against these decreases (i.e. caused relative increases compared with Familiarity), suggesting that it may buffer against decreases in compassion during repeated exposures to suffering. We tested for a neural parallel of these behavioral effects.

In 5 -fold cross-validated analyses, we selected the $5 \%$ of voxels exhibiting the largest pre-to-post-intervention decreases within a training subset of Familiarity participants. We then extracted the mean pre-to-post-intervention change in those voxels from the held-out participants, and we computed the standardized mean difference (Hedge's g) between Familiarity and CM participants in pre-to-post-intervention change scores. This provided a minimally biased procedure for identifying the largest neural decreases in the Familiarity group and testing for a buffering effect of CM in independent, held-out participants. Results were compared with a null distribution created by permuting the group labels and repeating the analysis 10000 times.

\section{Results}

\section{Behavioral results}

We first summarize the previously published (Ashar et al., 2016b) behavioral outcomes from the trial, to provide a context for the fMRI outcomes that are the focus of this manuscript.

Participant compliance, attention to task and expectations. Intervention compliance, as logged by the intervention iPod applications, was high across groups. At the same time, CM participants completed their daily tasks significantly less frequently than other participants (out of 28 possible days, CM: $M=20.76$ days, $95 \%$ CI $[18.81,22.71]$; OxyPla: $M=26.78$ days, 95\% CI [25.38, 28.18]; Familiarity: $M=25.39$ days, 95\% CI [24.10, 26.68]; $F(2,53)=17.15, P<0.001)$. Similarly, performance on the daily attention-to-task questions was near ceiling across groups,

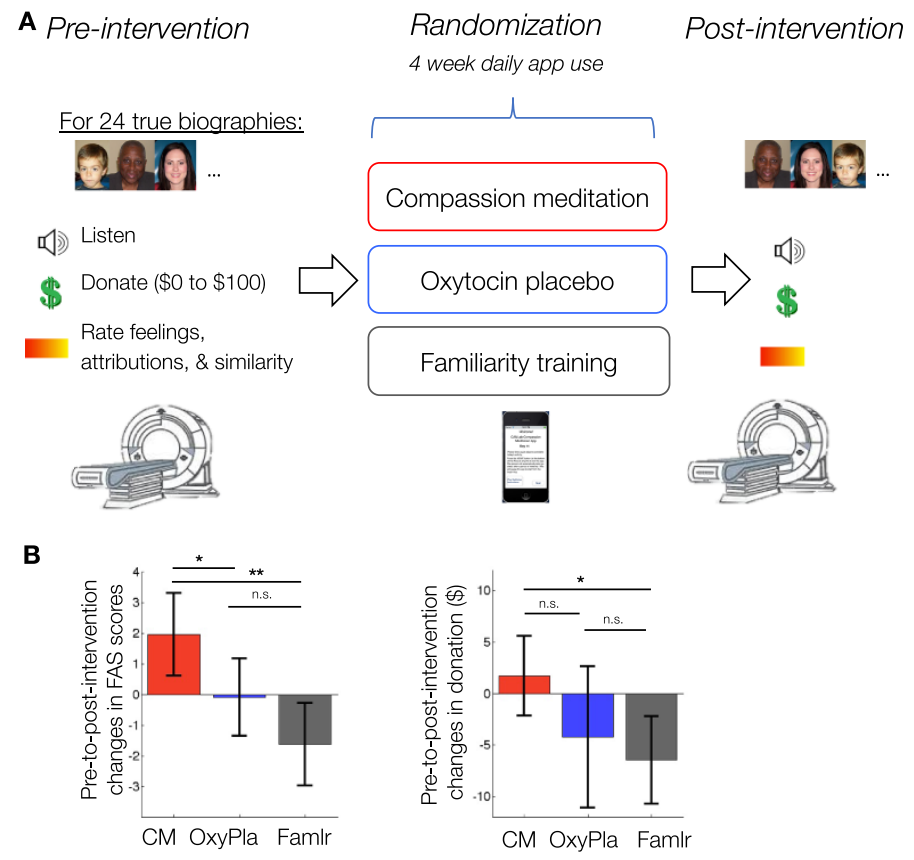

Fig. 1. Study design and behavioral results. (A) Participants completed a compassion task during functional MRI, were randomized to a compassion meditation intervention or a control intervention and then returned for a second functional MRI session. (B) Pre-to-post-intervention changes in charitable donations and in a composite index of compassion-related feelings and attributions ('FAS scores', see main text), as previously reported (Ashar et al., 2016b). Error bars show 95\% CI; ${ }^{*} \mathrm{P}<0.05,{ }^{* *} \mathrm{P}<0.01,{ }^{* * *} \mathrm{P}<0.001$. 
$M=98 \%$ correct, although there were significant group differences in correct responding, $F(2,54)=6.00, P=0.004$, which were driven by slightly lower performance in the CM group, $\mathrm{M}_{\mathrm{CM}}=95 \%$ correct, $95 \% \mathrm{CI}=[0.93,0.98]$.

Pre-intervention expectations of increased compassion were moderately high across groups, $M=5.00$ on a 0 to 10 scale, $95 \%$ CI $[4.40,5.60]$. There were significant group differences between the three groups, $F(2,49)=6.06, P=0.004$. This was driven by lower expectations in the Familiarity condition, $M=3.69$ out of 10, 95\% CI $[2.49,4.88]$. Expectations in the Familiarity condition were statistically lower relative to both the CM condition, $\mathrm{T}(32)=3.28, P=0.003,95 \% \mathrm{CI}[0.86,3.66]$, and the OxyPla condition, $\mathrm{T}(31)=1.97, P=0.06,95 \% \mathrm{CI}[-0.05,2.91]$. Direct comparison of expectations in the $\mathrm{CM}$ and OxyPla conditions showed no statistical difference, $T(33)=1.32, P=0.19$. At the study end, all but one of the OxyPla participants reported believing that they had been taking true oxytocin.

Changes in compassion and donation. Relative to Familiarity participants, CM participants increased in both FAS scores and charitable donations from pre- to post-intervention (Hedge's $g_{\mathrm{FAS}}=1.17,95 \%$ CI $[0.69,1.76]$, and $g_{\text {donation }}=0.89,95 \%$ CI $[0.35$, $1.47], M_{\text {donation difference }}=\$ 8.17,95 \%$ CI [ $\left.\left.\$ 2.22, \$ 14.11\right]\right)$. Relative to OxyPla participants, CM participants significantly increased in FAS scores, $g_{\mathrm{FAS}}=0.69,95 \% \mathrm{CI}[0.10,1.34]$, but the difference in donations was not statistically significant, $g_{\text {donation }}=0.48,95 \%$ CI $[-0.14,0.97], M_{\text {donation difference }}=\$ 5.95,95 \%$ CI $[\$-2.33, \$ 14.23]$. Examining patterns of absolute pre-to-post-intervention change within group, we found that CM participants increased in FAS scores, $g=0.61,95 \%$ CI $[0.27,1.03]$, and did not statistically change in donation amounts, $g=0.19,95 \%$ CI $[-0.27,0.60]$. OxyPla participants did not statistically change in either outcome, $g_{\mathrm{FAS}}=-0.03,95 \%$ CI $[-0.56,0.41], g_{\text {donation }}=-0.27,95 \%$ CI $[-0.58,0.22]$. Familiarity participants decreased in both outcomes, $g_{\mathrm{FAS}}=-0.53,95 \% \mathrm{CI}[-0.95,-0.18], g_{\text {donation }}=-0.67,95 \%$
CI [-1.06, -0.36] (Figure 1B). Overall, this profile of behavioral results suggests that (i) Familiarity participants decreased in compassion over time, (ii) CM buffered against this decrease and led to increased compassion and (iii) the effects of CM were partly but not fully attributable to placebo.

\section{fMRI results}

Region of Interest (ROI) analyses. For the CM us Familiarity comparison, we observed significantly increased brain responses to suffering others in the MOFC, $P=0.03$ FWERcorrected (Figure 2A, region center x y $z=[-1812-22] \mathrm{mm}$ in the MNI space). Individual differences in pre-to-post-intervention increases in mOFC activity were positively correlated with increases in FAS scores, $r(19)=0.50, P=0.04$ (Figure $2 C$ ), and not with charitable donations, $r(19)=0.28, P>0.2$. No CM us Familiarity group differences were observed in the dmPFC or TPJ. No $\mathrm{CM}$ us OxyPla group differences were observed, FWER-corrected within the regions of interest.

Whole-brain gray matter analyses. In whole-brain analyses applying an exploratory threshold, the CM us Familiarity comparison yielded three clusters with relatively increased activity for the CM condition: the mOFC, left superior temporal sulcus and left parahippocampal cortex. The CM us OxyPla comparison yielded three clusters with relatively increased activity in the CM condition: the MOFC and two occipital areas (Figure 2, coordinates listed in Supplementary Table S1).

For within-group pre-to-post-intervention changes, CM participants exhibited increased brain responses to suffering others in the mOFC, MPFC, midbrain and the right cerebellum. OxyPla participants exhibited no significant increases in brain activity over time and some decreases in V3 and lateral prefrontal areas. Familiarity participants exhibited decreases across several prefrontal and subcortical structures, including mOFC,

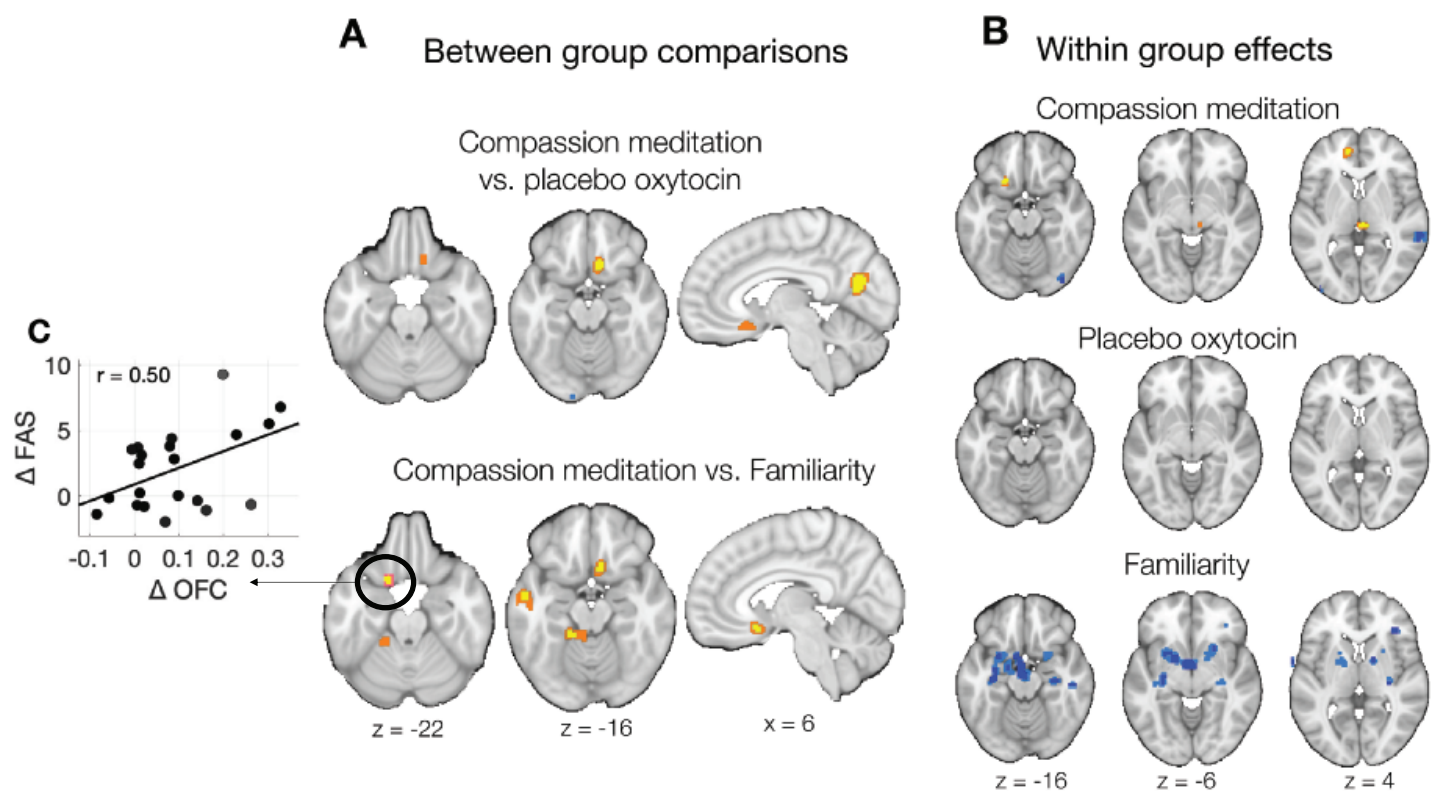

Fig. 2. Effects of CM us controls on brain responses to audio-video narratives describing suffering others. (A) Group differences in pre-to-post-intervention changes. (B) Absolute pre-to-post-intervention changes within each intervention condition. Circled clusters are significant at a threshold of $P<0.05$ FWER-corrected. Non-circled clusters meet an exploratory threshold of $\mathrm{P}<0.001$ uncorrected with 10 contiguous voxels. Adjacent voxels meeting a threshold of $\mathrm{P}<0.005$ uncorrected shown in orange/light blue for visualization purposes. Yellow/orange areas show increases and blue areas show decreases. (C) Among CM participants, increases in mOFC activity positively correlated with increased FAS scores, our primary measure of compassion-related emotions and appraisals (e.g. reduced blame and increased tenderness; see text for details). 
insula, superior temporal sulcus, amygdala, hippocampus, hypothalamus, putamen and globus pallidus (Figure 2B, coordinates listed in Supplementary Table S2).

Three-dimensional interactive images of all results are available at https://neurovault.org/collections/4766/, and figures showing full-brain results are provided in Supplementary Figures S1-S6.

Effects on a priori brain models of empathic care and distress. Effects of the intervention were in the expected direction, with CM participants exhibiting increases in the neural signature response (i.e. cosine similarity or pattern expression) for both empathic care and empathic distress relative to both OxyPla and Familiarity participants. However, group differences were not statistically significant, Ps ranging from 0.12 to 0.72 .

Tests of CM protecting against decreased brain responses to suffering others. We found a small 'buffering' effect of CM us Familiarity in the expected direction, $g=0.22$, which did not achieve statistical significance, $P>0.4$. Although pre-to-postintervention decreases across a range of subcortical regions were observed in Familiarity participants (Figure 2B), it is unclear to what extent CM protected against these.

Post-hoc power analyses. We observed an effect of magnitude $d=0.6$ in the mOFC for the CM us OxyPla comparison. Posthoc analyses revealed that a sample size of $N=45$ per group is needed for $80 \%$ power for an effect of this size, with two-tailed $\alpha=0.05$, indicating that future studies with resources for larger samples are needed.

\section{Discussion}

$\mathrm{CM}$ is a promising intervention for enhancing compassionate responding to suffering others. Here, we investigated the neurobiological mechanisms supporting the effects of CM. We compared a 4-week smartphone application-based CM intervention with two control conditions-placebo oxytocin (OxyPla) and a Familiarity control-to more closely isolate the specific effects of $\mathrm{CM}$ on brain function. We found significant CM Us Familiarity increases in mOFC responses to audiovisual narratives depicting suffering others, with CM us OxyPla effects on mOFC function also observed at an exploratory threshold. Among CM participants, individual differences in mOFC increases positively correlated with increases in compassion-related feelings and attributions (e.g. increased tenderness and reduced blame). We note that the mOFC region is adjacent to and potentially functionally overlaps with other anatomical labels, including subcallosal anterior cingulate. This area is also often included in the ventromedial prefrontal cortex (vmPFC), a broad zone encompassing multiple cortical regions (Koban et al., 2021).

Much research across humans and other species has strongly linked the mOFC-and larger, overlapping vmPFC zone-to a variety of forms of motivated behavior (Haber and Knutson, 2010), particularly when motivated behavior requires the construction of cognitive frames or schemas that supply personal meaning to events and actions (Roy et al., 2012; Barrett, 2017; Ashar et al., 2017b). In the interpersonal context, mOFC activity has been linked to empathic care for suffering others (Ashar et al., 2017a), altruistic giving (Cutler and Campbell-meiklejohn, 2019) and the perceived value of others (Watson and Platt, 2012; Zerubavel et al., 2015; Parkinson et al., 2017; Morelli et al., 2018).
Damage to the vmPFC and OFC is associated with increased utilitarian moral judgments (Koenigs et al., 2007), and pathological lack of empathy, guilt and remorse is associated with reduced vmPFC and OFC responses to stimuli depicting suffering others (Decety et al., 2013). Medial prefrontal representations also shift based on active perspective-taking processes, such that others' preferences are represented in more ventral MPFC areas when choosing on their behalf (Nicolle et al., 2012). Taken together, the engagement of the mOFC by CM may reflect an increased value placed on the welfare of suffering others, increased affiliation and/or increased empathic care. Integrative theories of vmPFCOFC function suggest that this is a conceptual process integrating information about another person with a representation of one's well-being.

Our findings are in broad agreement with a number of previous CM findings. Engen and Singer (2015b) found that practicing $\mathrm{CM}$ during viewing of emotional videos activates the mOFC, as compared with practicing cognitive reappraisal strategies. Randomized trials that compared CM with empathy and memory training have found that $\mathrm{CM}$ increases activity in mOFC areas overlapping or adjacent to the mOFC cluster reported here (Klimecki et al., 2012, 2014). Further, long-term meditators more strongly engage the mOFC when practicing CM relative to novice practitioners (Engen et al., 2018). Our findings are encouraging for the generalizability of the mOFC as an important neurobiological mechanism of CM. Relative to prior studies, ours tested a different $\mathrm{CM}$ implementation (including different specific CM practices, different delivery format and different intervention length) in a different participant population (participants from Germany us the USA). Since we did not ask CM participants to engage in CM practices during the scan, our findings also suggest that CM effects likely transfer to non-meditative states.

A recent debate has focused on whether CM primarily engages more cognitive or affective processes (Dahl et al., 2015, 2016; Engen and Singer, 2015a). Yet, cognitive and affective processes interact to promote empathy and compassion-both are important (De Waal, 2008; Zaki and Ochsner, 2012; Kanske et al., 2016). While mOFC function has been typically more closely related to affective than cognitive empathy processes, both these processes are likely intertwined in the mOFC (Roy et al., 2012; Barrett, 2017; Ashar et al., 2017b). Relatedly, our results cannot disambiguate between processes that are activated during the practice of CM us the outcome of CM (Dahl et al., 2016). CM also refers to a family of meditation practices, and there may be substantial variability in mechanisms among different CM implementations, with some engaging more cognitive processes. For example, cognitively based compassion training uses a 'cognitive, analytic approach to challenge one's unexamined thoughts and emotions toward other people... various arguments are examined that challenge one's common sense notion of other people as falling into the categories of "friend, enemy and stranger"' (Pace et al., 2009).

What are the specific effects of $\mathrm{CM}$ on brain function, above and beyond placebo and familiarity effects? We found a significant effect of CM us Familiarity in mOFC responses to suffering others, with weaker CM vs OxyPla effects observed at an exploratory threshold. These results parallel the selfreported and behavioral outcomes, in which we observed significant group differences for the CM us Familiarity comparison, which were attenuated for the CM us OxyPla comparison. Overall, these findings indicate effects of CM on brain function are not explained by familiarity effects and are partly explained by placebo effects. Another investigation comparing mindfulness meditation to sham (placebo) meditation similarly reported 
specific effects of mindfulness on brain function, in this case, in responses to painful experimental stimuli (Zeidan et al., 2010, 2015).

A critical meditation research challenge is developing wellmatched control conditions (MacCoon et al., 2012; Davidson and Kaszniak, 2015). Our placebo manipulation succeeded in generating pre-intervention expectations of increased compassion similar to those reported in the CM group, and all but one participant reported believing they had been taking verum oxytocin. At the same time, the placebo oxytocin nasal spray likely engaged an overlapping but distinct set of expectations and associations relative to CM. For example, oxytocin is often portrayed as the 'love hormone' in popular culture-a reference related to but different from compassion-and participants might have expected different time courses of intervention effects from nasal spray us meditation. Designing controls with tightly matched expectations is a major challenge for psychological interventions (Boot et al., 2013).

Additionally, the two CM comparison conditions included here were not matched on other non-specific factors, such as time spent engaged in the intervention each day. CM participants completed the CM intervention less often than the control interventions (although CM compliance was still relatively high overall-encouraging for a smartphone CM implementation). This relatively less-frequent engagement with the CM app may have either detracted from its efficacy (due to a lower 'dose' of $\mathrm{CM}$ ) or enhanced it (if more exposure to suffering reduces compassion). Future studies including more closely matched control groups will continue to refine our understanding of the 'active ingredients' of CM.

We cannot generalize our findings to participants who are unwilling to donate to charity, as these subjects were excluded. It is unknown whether these people differ in their response to $\mathrm{CM}$ interventions. Our results also have limited generalizability to groups underrepresented in our sample, including racial/ethnic minorities. Racial/ethnic minority groups have been broadly underrepresented in meditation research (Waldron et al., 2018), marking an important area for future research.

We observed decreased activity in Familiarity participants across a range of prefrontal and subcortical regions, including the mOFC, amygdala, hippocampus, insula and more. These decreases were not observed in the CM and/or placebo groups, suggesting a potential 'buffering effect' of these interventions against decreased compassion, as seen in the behavioral data. However, we did not detect a significant group by time interaction in these regions (besides in the mOFC), and direct tests of this buffering hypothesis failed to reach statistical significance. Alternately, reduced responsivity to others' suffering might be a positive outcome in some ways and in some contexts, since heightened emotional responses to suffering can lead to professional 'burnout' (Maslach et al., 2001; Shanafelt et al., 2017). In support of this, mindfulness-based interventions have been found to reduce brain responses to emotionally distressing stimuli, which was interpreted as a positive outcome (Desbordes et al., 2012). Future studies are needed to better understand how reduced brain responses to suffering others may alternately reflect equanimity or diminished compassion.

Our exploratory analyses additionally revealed several additional regions of potential interest. As these regions did not survive correction for multiple comparisons, further studies might conduct more targeted investigations of their role in $\mathrm{CM}$. One finding of interest is the increased response in visual cortex for
CM us OxyPla, which might reflect increased visual engagement with the stimuli depicting suffering others. A second finding of interest is the increased parahippocampal response for CM us Familiarity, which might reflect increased recruitment of memory- or imagination-related processes during engagement with suffering others, consistent with recent work on the importance of the episodic memory system for compassion (Gaesser et al., 2019a,b).

In sum, our findings suggest that compassion training can increase psychological, behavioral and brain processes that support empathic care and prosocial behavior. Compassion training rests of the assumption that compassion is a skill and a choice (Halifax, 2012; Zaki, 2014). Studies have found that it is often a difficult choice-engaging with others' suffering is effortful and often avoided (Cameron, 2017; Cameron et al., 2019). Yet, compassion training programs can teach skills for engaging with others' suffering without becoming overwhelmed, cultivating a sensitive and sustainable approach for enacting compassion in daily lives. Investigating the psychological and neurobiological mechanisms of compassion training interventions will help us better understand these processes, promoting their development and dissemination.

\section{Acknowledgements}

We express our gratitude to research assistants Jenifer Mutari, Robin Kay, Scott Meyers, Nicholas Peterson and Brandin Williams for their help with data collection.

\section{Funding}

This work was funded by the John Templeton Foundation's Positive Neuroscience project (PIs Wager and Dimidjian), with additional support from NIH R01 R01DA035484 (PI Wager) and NIH/NCATS Grant \# TL1-TR-002386 (PI Ashar).

\section{Conflicts of interest}

None declared.

\section{Author contributions}

T.D.W and S.D. developed the study concept. All authors contributed to the study design. Testing and data collection were performed by Y.K.A. and research assistants under the supervision of Y.K.A. Data analysis and interpretation were conducted by Y.K.A. under the supervision of T.D.W. Y.K.A drafted the manuscript. All authors provided critical revisions and approved the final version of the manuscript for submission.

\section{Supplementary data}

Supplementary data are available at SCAN online.

\section{References}

Adler, N., Epel, E., Castellazzo, G., Ickovics, J. (2000). Relationship of subjective and objective social status with psychological and physiological functioning: preliminary data in healthy, white women. Health Psychology, 19(6), 586-92. http:// psycnet.apa.org/journals/hea/19/6/586/. 
Amunts, K., Mohlberg, H., Bludau, S., Zilles, K. (2020). JulichBrain: a 3D probabilistic atlas of the human brain's cytoarchitecture. Science, 369(6506), 988-92.

Ashar, Y.K., Andrews-Hanna, J.R., Dimidjian, S., Wager, T.D. (2016a). Towards a neuroscience of compassion: a brain systems-based model and research agenda. In: Greene, J.D., editor. Positive Neuroscience, New York: Oxford University Press, $1-27$.

Ashar, Y.K., Andrews-Hanna, J.R., Yarkoni, T., et al. (2016b). Effects of compassion meditation on a psychological model of charitable donation. Emotion, 16(5), 691-705.

Ashar, Y.K., Andrews-Hanna, J.R., Dimidjian, S., Wager, T.D. (2017a). Empathic care and distress: predictive brain markers and dissociable brain systems. Neuron, 94(6), 1-11.

Ashar, Y.K., Chang, L.J., Wager, T.D. (2017b). Brain mechanisms of the placebo effect: an affective appraisal account. Annual Review of Clinical Psychology, 13(1), 73-98.

Barrett, L.F. (2017). The theory of constructed emotion: an active inference account of interoception and categorization. Social Cognitive and Affective Neuroscience, 12(1), 1-23.

Berridge, K.C., Kringelbach, M.L. (2015). Pleasure systems in the brain. Neuron, 86(3), 646-64.

Berry, M.P., Lutz, J., Schuman-Olivier, Z., et al. (2020). Brief selfcompassion training alters neural responses to evoked pain for chronic low back pain: a pilot study. Pain Medicine, 21(10), $1-14$.

Böckler, A., Tusche, A., Schmidt, P., Singer, T. (2018). Distinct mental trainings differentially affect altruistically motivated, norm motivated, and self-reported prosocial behaviour. Scientific Reports, 8(1), 13560.

Boot, W.R., Simons, D.J., Stothart, C., Stutts, C. (2013). The pervasive problem with placebos in psychology: why active control groups are not sufficient to rule out placebo effects. Perspectives on Psychological Science, 8(4), 445-54.

Bornemann, B., Kok, B.E., Böckler, A., Singer, T. (2016). Helping from the heart: voluntary upregulation of heart rate variability predicts altruistic behavior. Biological Psychology, 119, 54-63.

Buckner, R.L., Carroll, D.C. (2007). Self-projection and the brain. Trends in Cognitive Sciences, 11(2), 49-57.

Cameron, D. (2017). Compassion collapse. Why we are numb to numbers. In: Sepalla, E., Simon-Thomas, E.R., Brown, S.L., Worline, M., Cameron, D.C., Doty, J.R., editors. The Oxford Handbook of Compassion Science. Oxford University Press, Vol. 1, $1-21$.

Cameron, D., Hutcherson, C., Ferguson, A., Andrew Scheffer, J., Hadjiandreou, E., Inzlicht, M. (2019). Empathy is hard work: people choose to avoid empathy because of its cognitive costs. Journal of Experimental Psychology: General, 148(6), 962-76.

Condon, P., Desbordes, G., Miller, W.B., DeSteno, D. (2013). Meditation increases compassionate responses to suffering. Psychological Science, 24(10), 2125-7.

Cutler, J., Campbell-meiklejohn, D. (2019). A comparative fMRI meta-analysis of altruistic and strategic decisions to give. NeuroImage, 184, 227-41.

Dahl, C.J., Lutz, A., Davidson, R.J. (2015). Reconstructing and deconstructing the self: cognitive mechanisms in meditation practice. Trends in Cognitive Sciences, 19(9), 515-23.

Dahl, C.J., Lutz, A., Davidson, R.J. (2016). Cognitive processes are central in compassion meditation. Trends in Cognitive Sciences, 20, 1-2.

Darnall, B.D. (2015). Compassion cultivation in chronic pain may reduce anger, pain, and increase acceptance: study review and brief commentary. Health Care Current Reviews, 3, 2.
Davidson, R.J., Kaszniak, A.W. (2015). Conceptual and methodological issues in research on mindfulness and meditation. American Psychologist, 70(7), 581-92.

de Waal, F.B.M. (2008). Putting the altruism back into altruism: the evolution of empathy. Annual Review of Psychology, 59, 279-300.

Decety, J., Norman, G.J., Berntson, G.G., Cacioppo, J.T. (2012). A neurobehavioral evolutionary perspective on the mechanisms underlying empathy. Progress in Neurobiology, 98(1), 38-48.

Decety, J., Skelly, L.R, Kiehl, K.A (2013). Brain response to empathy-eliciting scenarios involving pain in incarcerated individuals with psychopathy. JAMA Psychiatry, 70(6), 638-45.

Desbordes, G., Negi, L.T., Pace, T.W.W., Wallace, B.A., Raison, C.L., Schwartz, E.L. (2012). Effects of mindful-attention and compassion meditation training on amygdala response to emotional stimuli in an ordinary, non-meditative state. Frontiers in Human Neuroscience, 6, 1-15.

Engen, H.G., Bernhardt, B.C., Skottnik, L., Ricard, M., Singer, T. (2018). Structural changes in socio-affective networks: multimodal MRI findings in long-term meditation practitioners. Neuropsychologia, 116, 26-33.

Engen, H.G., Singer, T. (2015a). Affect and motivation are critical in constructive meditation. Trends in Cognitive Sciences, 20, 1-2.

Engen, H.G., Singer, T. (2015b). Compassion-based emotion regulation up-regulates experienced positive affect and associated neural networks. Social Cognitive and Affective Neuroscience, 10(9), 1291-301.

Favre, P., Kanske, P., Engen, H., Singer, T. (2020). Decreased emotional reactivity after 3-month socio-affective but not attention- or meta-cognitive-based mental training: a randomized, controlled fMRI study. PsyArXiv, 33, 1-47.

Gaesser, B., Hirschfeld-Kroen, J., Wasserman, E.A., Horn, M., Young, L. (2019a). A role for the medial temporal lobe subsystem in guiding prosociality: the effect of episodic processes on willingness to help others. Social Cognitive and Affective Neuroscience, 14(4), 397-410.

Gaesser, B., Shimura, Y., Cikara, M., Gaesser, B., Cikara, M. (2019b). Prosocial intentions and behavior episodic simulation reduces intergroup bias in prosocial intentions and behavior. Journal of Personality and Social Psychology, 118(4), 683.

Galante, J., Galante, I., Bekkers, M., Gallacher, J. (2014). Effect of kindness-based meditation on health and well-being: a systematic review and meta-analysis. Journal of Consulting and Clinical Psychology, 82(6), 1101-14. http://psycnet.apa.org/ psycinfo/2014-26574-001/.

Gilbert, P. (2014). The origins and nature of compassion focused therapy. British Journal of Clinical Psychology, 53(1), 6-41.

Glasser, M.F., Coalson, T.S., Robinson, E.C., et al. (2016). A multi-modal parcellation of human cerebral cortex. Nature, 536(7615), 1-11.

Goetz, J.L., Keltner, D., Simon-Thomas, E. (2010). Compassion: an evolutionary analysis and empirical review. Psychological Bulletin, 136(3), 351-74.

Haber, S.N., Knutson, B. (2010). The reward circuit: linking primate anatomy and human imaging. Neuropsychopharmacology, 35(1), 4-26.

Halifax, J. (2011). The precious necessity of compassion. Journal of Pain and Symptom Management, 41(1), 146-153.

Halifax, J. (2012). A heuristic model of enactive compassion. Current Opinion in Supportive and Palliative Care, 6(2), 228-35.

Hare, T.A., Camerer, C.F., Knoepfle, D.T., Rangel, A. (2010). Value computations in ventral medial prefrontal cortex during charitable decision making incorporate input from regions 
involved in social cognition. The Journal of Neuroscience: The Official Journal of the Society for Neuroscience, 30(2), 583-90.

Hofmann, S.G., Grossman, P., Hinton, D.E. (2011). Lovingkindness and compassion meditation: potential for psychological interventions. Clinical Psychology Review, 31(7), 1126-32.

Inagaki, T.K., Eisenberger, N.I. (2012). Neural correlates of giving support to a loved one. Psychosomatic Medicine, 74(1), 3-7.

Kanske, P., Bockler, A., Trautwein, F.M., Lesemann, F.H.P., Singer, T. (2016). Are strong empathizers better mentalizers? Evidence for independence and interaction between the routes of social cognition. Social Cognitive and Affective Neuroscience, 11(9), 1383-92.

Klimecki, O.M., Leiberg, S., Lamm, C., Singer, T. (2012). Functional neural plasticity and associated changes in positive affect after compassion training. Cerebral Cortex, 23, 1-10.

Klimecki, O.M., Leiberg, S., Ricard, M., Singer, T. (2014). Differential pattern of functional brain plasticity after compassion and empathy training. Social Cognitive and Affective Neuroscience, 9(6), 873-9.

Koban, L., Gianaros, P.J., Kober, H., Wager, T.D. (2021). The self in context: brain systems linking mental and physical health. Nature Review Neuroscience, 22, 309-22.

Koenigs, M., Young, L., Adolphs, R., et al. (2007). Damage to the prefrontal cortex increases utilitarian moral judgements. Nature, 446(7138), 908-11.

Kok, B.E., Singer, T. (2016). Effects of contemplative dyads on engagement and perceived social connectedness over 9 months of mental training. JAMA Psychiatry, 199(1-2), 23-9.

Kok, B.E., Singer, T. (2017). Phenomenological fingerprints of four meditations: differential state changes in affect, mindwandering, meta-cognition, and interoception before and after daily practice across 9 months of training. Mindfulness, 8(1), 218-31.

Koopmann-Holm, B., Sze, J., Jinpa, T., Tsai, J.L. (2020). Compassion meditation increases optimism towards a transgressor. Cognition and Emotion, 34(5), 1028-35.

Kragel, P.A., Kano, M., Van Oudenhove, L., et al. (2018). Generalizable representations of pain, cognitive control, and negative emotion in medial frontal cortex. Nature Neuroscience, 21(2), 283-9.

Kreplin, U., Farias, M., Brazil, I.A. (2018). The limited prosocial effects of meditation: a systematic review and meta-analysis. Scientific Reports, 8(1), 1-10.

Krienen, F.M., Tu, P.-C., Buckner, R.L. (2010). Clan mentality: evidence that the medial prefrontal cortex responds to close others. Journal of Neuroscience, 30(41), 13906-15.

Lieberman, M.D., Cunningham, W.A. (2009). Type I and Type II error concerns in fMRI research: re-balancing the scale. Social Cognitive and Affective Neuroscience, 4(4), 423-8.

Lim, D., Condon, P., de Steno, D. (2015). Mindfulness and compassion: an examination of mechanism and scalability. PLoS One, 10(2), 1-8.

Loewenstein, G., Small, D.A. (2007). The Scarecrow and the Tin Man: the vicissitudes of human sympathy and caring. Review of General Psychology, 11(2), 112-26.

Lutz, A., Brefczynski-Lewis, J., Johnstone, T., Davidson, R.J. (2008). Regulation of the neural circuitry of emotion by compassion meditation: effects of meditative expertise. PLoS One, 3(3), e1897.

MacCoon, D.G., Imel, Z.E., Rosenkranz, M.A., et al. (2012). The validation of an active control intervention for Mindfulness Based Stress Reduction (MBSR). Behaviour Research and Therapy, 50(1), 3-12.
Mascaro, J.S., Rilling, J.J.K., Tenzin Negi, L., Raison, C.L. (2013). Compassion meditation enhances empathic accuracy and related neural activity. Social Cognitive and Affective Neuroscience, 8(1), 48-55.

Maslach, C., Schaufeli, W.B., Leiter, M.P. (2001). Job burnout. Annual Review Psychology, 52, 397-422.

Meyer, M.L., Masten, C.L., Ma, Y., et al. (2013). Empathy for the social suffering of friends and strangers recruits distinct patterns of brain activation. Social Cognitive and Affective Neuroscience, $8(4), 446-54$.

Mitchell, T.M., Shinkareva, S.V., Carlson, A., et al. (2008). Predicting human brain activity associated with the meanings of nouns supplement. Science, 320(5880), 1191-5.

Mobbs, D., Yu, R., Meyer, M., et al. (2009). A key role for similarity in vicarious reward. Science, 324(5929), 900.

Moll, J., Weingartner, J.H., Bado, P., et al. (2014). Voluntary enhancement of neural signatures of affiliative emotion using fMRI neurofeedback. PLoS One, 9(5), e97343.

Morelli, S.A., Sacchet, M.D., Zaki, J. (2015). Common and distinct neural correlates of personal and vicarious reward: a quantitative meta-analysis. NeuroImage, 112, 244-53.

Morelli, S.A., Leong, Y.C., Carlson, R.W., Kullar, M., Zaki, J. (2018). Neural detection of socially valued community members. Proceedings of the National Academy of Sciences of the United States of America, 115(32), 8149-54.

Nicolle, A., Klein-Flügge, M.C., Hunt, L.T., Vlaev, I., Dolan, R.J., Behrens, T.E.J. (2012). An agent independent axis for executed and modeled choice in medial prefrontal cortex. Neuron, 75(6), 1114-21.

Nook, E.C., Ong, D.C., Morelli, S.A., Mitchell, J.P., Zaki, J. (2016). Prosocial conformity: prosocial norms generalize across behavior and empathy. Personality and Social Psychology Bulletin, 42(8), 1045-62.

Pace, T.W.W., Negi, L.T., Adame, D.D., et al. (2009). Effect of compassion meditation on neuroendocrine, innate immune and behavioral responses to psychosocial stress. Psychoneuroendocrinology, 34(1), 87-98.

Pace, T.W.W., Tenzin, L., Dodson-lavelle, B., et al. (2012). Engagement with cognitively-based compassion training is associated with reduced salivary C-reactive protein from before to after training in foster care program adolescents. Psychoneuroendocrinology, 38(2), 294-99.

Parkinson, C., Kleinbaum, A.M., Wheatley, T. (2017). Spontaneous neural encoding of social network position. Nature Human Behaviour, 1(5), 1-7.

Pauli, W.M., Nili, A.N., Tyszka, J.M. (2018). A high-resolution probabilistic in vivo atlas of human subcortical brain nuclei. Scientific Data, 5, 180063.

Quaglia, J.T., Soisson, A., Simmer-Brown, J. (2020). Compassion for self versus other: a critical review of compassion training research. Journal of Positive Psychology, 1-16.

Roy, M., Shohamy, D., Wager, T.D. (2012). Ventromedial prefrontal-subcortical systems and the generation of affective meaning. Trends in Cognitive Sciences, 16(3), 147-56.

Rutgen, M., Seidel, E.-M., Rie Ansky, I., Lamm, C. (2015). Reduction of empathy for pain by placebo analgesia suggests functional equivalence of empathy and first-hand emotion experience. Journal of Neuroscience, 35(23), 8938-47.

Rütgen, M., Seidel, E.-M., Silani, G., et al. (2015). Placebo analgesia and its opioidergic regulation suggest that empathy for pain is grounded in self pain. Proceedings of the National Academy of Sciences of the United States of America, 112(41), E5638-46. 
Saxe, R., Kanwisher, N. (2003). People thinking about thinking people. The role of the temporo-parietal junction in "theory of mind". NeuroImage, 19(4), 1835-42.

Segal, Z.V., Bieling, P., Young, T., et al. (2010). Antidepressant monotherapy vs sequential pharmacotherapy and mindfulness-based cognitive therapy, or placebo, for relapse prophylaxis in recurrent depression. Archives of General Psychiatry, 67(12), 1256-64.

Shamay-Tsoory, S.G., Aharon-Peretz, J., Perry, D. (2009). Two systems for empathy: a double dissociation between emotional and cognitive empathy in inferior frontal gyrus versus ventromedial prefrontal lesions. Brain: A Journal of Neurology, 132(Pt 3), 617-27.

Shanafelt, T., Dyrbye, L., West, C. (2017). Addressing physician burnout: the way forward. JAMA, 317(9), 901-2.

Shonin, E., Van Gordon, W., Compare, A., Zangeneh, M., Griffiths, M.D. (2015). Buddhist-derived loving-kindness and compassion meditation for the treatment of psychopathology: a systematic review. Mindfulness, 6(5), 1161-80.

Singer, T., Engert, V. (2019). It matters what you practice: differential training effects on subjective experience, behavior, brain and body in the resource project. Current Opinion in Psychology, 28, 151-8.

Singer, T., Klimecki, O.M. (2014). Empathy and compassion. Current Biology: $C B, 24(18)$, R875-8.

Sirotina, U., Shchebetenko, S. (2020). Loving-kindness meditation and compassion meditation: do they affect emotions in a different way? Mindfulness, 11(11), 2519-30.

Smith, S.M., Nichols, T.E. (2009). Threshold-free cluster enhancement: addressing problems of smoothing, threshold dependence and localisation in cluster inference. NeuroImage, 44(1), 83-98.

Tankersley, D., Stowe, C.J., Huettel, S. (2007). Altruism is associated with an increased neural response to agency. Nature Neuroscience, 10(2), 150-1.

Valk, S.L., Bernhardt, B.C., Trautwein, F.M., et al. (2017). Structural plasticity of the social brain: differential change after socioaffective and cognitive mental training. Science Advances, 3(10), $1-12$.

van Berkhout, E.T., Malouff, J.M. (2015). The efficacy of empathy training: a meta-analysis of randomized controlled trials. Journal of Counseling Psychology, 63(1), 32-41.

Wager, T.D., Keller, M.C., Lacey, S.C., Jonides, J. (2005). Increased sensitivity in neuroimaging analyses using robust regression. NeuroImage, 26(1), 99-113.

Waldron, E.M., Hong, S., Moskowitz, J.T., Burnett-Zeigler, I. (2018). A systematic review of the demographic characteristics of participants in US-based randomized controlled trials of mindfulness-based interventions. Mindfulness, 9(6), 1671-92.

Watson, K.K., Platt, M.L. (2012). Social signals in primate orbitofrontal cortex. Current Biology, 22(23), 2268-73.

Weng, H.Y., Fox, A.S., Shackman, A.J., et al. (2013). Compassion training alters altruism and neural responses to suffering. Psychological Science, 24(7), 1171-80.

Weng, H.Y., Fox, A.S., Hessenthaler, H.C., Stodola, D.E., Davidson, R.J. (2015). The role of compassion in altruistic helping and punishment behavior. PLoS One, 10(12), e0143794.

Weng, H.Y., Schuyler, B., Davidson, R.J. (2017). The Impact of Compassion Meditation Training on the Brain and Prosocial Behavior, The Oxford Handbook of Compassion Science. Oxford University Press. 133-46.

Williams, J.M.G., Crane, C., Barnhofer, T., et al. (2014). Mindfulness-based cognitive therapy for preventing relapse in recurrent depression: a randomized dismantling trial. Journal of Consulting and Clinical Psychology, 82(2), 275-86.

Woo, C.W., Krishnan, A., Wager, T.D. (2014). Cluster-extent based thresholding in fMRI analyses: pitfalls and recommendations. NeuroImage, 91, 412-9.

Zajonc, R.B. (2001). Mere exposure: a gateway to the subliminal. Current Directions in Psychological Science, 10(6), 224-8.

Zaki, J. (2014). Empathy: a motivated account. Psychological Bulletin, 140(6), 1608-47. http://psycnet.apa.org/journals/bul/140/ $6 / 1608 /$.

Zaki, J., Ochsner, K. (2012). The neuroscience of empathy: progress, pitfalls and promise. Nature Neuroscience, 15(5), 675-80.

Zeidan, F., Johnson, S.K., Gordon, N.S., Goolkasian, P. (2010). Effects of brief and sham mindfulness meditation on mood and cardiovascular variables. Journal of Alternative and Complementary Medicine, 16(8), 867-73.

Zeidan, F., Emerson, N.M., Farris, S.R., et al. (2015). Mindfulness meditation-based pain relief employs different neural mechanisms than placebo and sham mindfulness meditation-induced analgesia. Journal of Neuroscience, 35(46), 15307-25.

Zerubavel, N., Bearman, P.S., Weber, J., Ochsner, K.N. (2015). Neural mechanisms tracking popularity in real-world social networks. Proceedings of the National Academy of Sciences of the United States of America, 112(49), 15072-7.

Zickfeld, J.H., Schubert, T.W., Seibt, B., et al. (2019). Kama muta: conceptualizing and measuring the experience often labelled being moved across 19 nations and 15 languages. Emotion, 19(3), 402-24. 